\title{
Compromise Mixed Allocation in Multivariate Stratified Sampling Using Dynamic Programming Technique
}

\author{
A. H. Ansari ${ }^{*}$, Rahul Varshney ${ }^{2}$ and M. J. Ahsan ${ }^{3}$ \\ ${ }^{1}$ Kejriwal Institute of Management and Development Studies, Namkum, Ranchi-834010, India \\ ${ }^{2}$ Department of Applied Statistics Babasaheb Bhimrao Ambedkar University, Lucknow 226025, India \\ ${ }^{3}$ Department of Statistics and Operations Research Aligarh Muslim University, Aligarh 202 002, India \\ ${ }^{1}$ Email: athar_hussain1@rediffmail.com \\ ${ }^{2}$ Email: itsrahulvarshney@gmail.com \\ ${ }^{3}$ Email: mjahsan2001@yahoo.co.in, Mob: 9897356448
}

\begin{abstract}
The idea of "Mixed Allocation" in stratified sampling was introduced by [4]. The concept was further developed by several authors in different manner. In the present paper the authors worked out the "Compromise Mixed Allocation" for multivariate stratified sampling for more than one. Say " $p$ " characteristics using Dynamic Programming Technique are defined on each population unit. It is assumed that the properties of the strata on which the grouping scheme of [4] is based are prevalent in the multivariate case also. Numerical examples are also presented to illustrate the computational details.
\end{abstract}

Keywords: Stratified sampling, optimum allocation, mixed allocation, multivariate stratified sampling, dynamic programming technique, compromise allocation, compromise mixed allocation, relative loss in efficiency.

\section{Introduction}

The literal meaning of the word "compromise" is "via media", but it has a special meaning in stratified sampling literature where different types of allocation procedure like Equal, proportional, optimum and several other allocations are present. Usually any one type of allocation is selected according to the nature of the population, the use of single allocation procedure is not advisable to all strata due to practical implications. In such situations, one can divide the strata into $k$ different groups which are non-overlapping and exhaustive groups that are similar in nature internally. A particular type of allocation can then be applied to a particular group of strata depending on the nature of the group. [4] worked out the allocation using the above criterion and named it as "Mixed Allocation".

They formulated the problem of finding a mixed allocation as the following nonlinear programming problem (NLPP)

$$
\begin{gathered}
\text { Minimize } \quad F\left(\alpha_{j}\right)=\sum_{j=1}^{k} \sum_{h \in I_{j}} \frac{W_{h}^{2} S_{h}^{2}}{\alpha_{j} \beta_{h}} \\
\text { subject to } \quad \sum_{j=1}^{k} \sum_{h \in I_{j}} \alpha_{j} c_{h} \beta_{h} \leq C_{0} \\
\text { and } \quad \alpha_{j} \geq 0 ; j=1,2, \ldots, k
\end{gathered}
$$

where $L$ strata are divided into $k$ groups, the $j^{\text {th }}$ group consists of $L_{j}$ strata. The sample allocations are given by

$$
n_{h}=\alpha_{j} \beta_{h} ; h \in I_{j}, j=1,2, \ldots, k
$$

where $\alpha_{j} ; j=1,2, \ldots, k$ are the solution to NLPP (1.1) - (1.3), $I_{j}$ is the set of integers representing the strata numbers in the $j^{\text {th }}$ group and $\beta_{h}$ is fixed according to the particular allocation used. For example

*Corresponding Author 
if proportional allocation is to be used in the $q^{\text {th }}$ group then $\beta_{h}=W_{h} ; h \in I_{q}$.

In multivariate stratified sampling where $p(>1)$ characteristics are to be measured on each selected unit of the sample, an allocation that minimizes the variance of one characteristic may result in significant losses of precision for other characteristics. For such situations [26] gave the idea of "Compromise Allocation" which minimizes the weighted sum of variances of all the p-characteristics for a fixed budget this type of allocation is based on a compromise criterion to have a combined objective instead of several objectives (minimizing the individual variances). After that different authors suggested different compromise criteria or explored further the already existing criteria. Among them are [26], [6], $[16],[20],[10],[11],[7],[2],[3],[24],[8],[9],[23],[17],[18],[19],[14],[15],[5]$ and many others. [21] discussed five different compromise criteria to work out approximate optimum allocation in multivariate surveys and compared them using a simulation study. [22] gave three different compromise criteria and modified the random search method to develop an algorithm to obtain the compromise allocation for multivariate stratified populations.

Before [4] no author used the term "Mixed Allocation" and thus no sampling literature is available on mixed allocation. However, [12] used a similar idea in univariate two-stage sampling design. In multivariate case instead of individual optimum allocations usually a compromise allocation is used.

[4] worked out the mixed allocation for univariate stratified sampling. In this paper we extended the work of [4] for the multivariate case using Dynamic Programming Technique (DPT). Thus the present paper presents a combination of Compromise and Mixed allocations. The allocation thus obtained, may be termed as "Compromise Mixed Allocation".

Section 2 gives the formulation and solution of the problem. Section 3 highlights the situation in which the compromise mixed allocation may be used in practice. Section 4 illustrates a numerical example to justify the use of compromise mixed allocation. Section 5 summarizes the comparative performance of the proposed allocation with some other compromise allocations. Section 6 gives the concluding remark on the basis of the results obtained in Sections 4 and 5 .

A list of alphabetically arranged references is provided at the end of the paper.

\section{Formulation}

Using [26] criterion the problem of finding the mixed allocation given in (1.1) - (1.3) for multivariate case may be expressed as

$$
\begin{gathered}
\text { Minimize } \sum_{l=1}^{p} a_{l} \sum_{j=1}^{k} \sum_{h \in I_{j}} \frac{W_{h}^{2} S_{l h}^{2}}{\alpha_{j} \beta_{h}} \\
\text { subject to } \sum_{j=1}^{k} \sum_{h \in I_{j}} \alpha_{j} c_{l h} \beta_{h} \leq C_{0} \\
\text { and } \quad \alpha_{j} \geq 0 ; j=1,2, \ldots, k, \quad C_{0}=C-c_{0}
\end{gathered}
$$

where $a_{l}>0$ is the weight assigned to the variance of the $l^{\text {th }}$ characteristic, $S_{l h}^{2}$ is the stratum variance for the $l^{\text {th }}$ characteristic, $C$ is the total budget, $c_{0}$ is the overhead cost, $C_{0}$ is the available budget after deducting the overhead cost and $n_{h}=\alpha_{j} \beta_{h}$. Here-in-after $c_{h}$ will denote the cost of measuring for all the $p$ characteristics on a selected unit of $h^{\text {th }}$ stratum, that is $c_{h}=\sum_{l=1}^{p} c_{l h}$ with $h=1,2, \ldots, L$, and $c_{l h}$ denoting the per unit cost of measurement for the $l^{\text {th }}$ characteristic in the $h^{\text {th }}$ stratum. Without loss of generality we can assume that $\sum_{l=1}^{p} a_{l}=1$.

Substituting $A_{h}=W_{h}^{2} \sum_{l=1}^{p} a_{l} S_{l h}^{2} ; h=1,2, \ldots, L$ and rearranging the terms NLPP (2.1) - (2.3) may be restated as

$$
\text { Minimize } \sum_{j=1}^{k} \sum_{h \in I_{j}} \frac{A_{h}}{\alpha_{j} \beta_{h}}
$$




$$
\begin{gathered}
\text { subject to } \quad \sum_{j=1}^{k} \sum_{h \in I_{j}} \alpha_{j} c_{h} \beta_{h} \leq C_{0} \\
\text { and } \quad \alpha_{j} \geq 0 ; j=1,2, \ldots, k
\end{gathered}
$$

Since the objective function is convex and the constraint is linear at the optimal point the constraint will be active [1]. The NLPP (2.4) - (2.6) may be solved by Dynamic Programming Technique.

$$
\begin{array}{ll}
\text { Minimize } & F\left(\alpha_{j}\right)=\sum_{j=1}^{k} \sum_{h \in I_{j}} \frac{A_{h}}{\alpha_{j} \beta_{h}} \\
\text { subject to } & \sum_{j=1}^{k} \sum_{h \in I_{j}} \alpha_{j} c_{h} \beta_{h} \leq C_{0} \\
\text { and } & \alpha_{i} \geq 0 ; j=1,2, \ldots, k
\end{array}
$$

Consider the $r^{\text {th }}$ stage sub problem of MPP (2.7) for the first $\mathrm{r}(<\mathrm{k})$ groups.

$$
\begin{array}{ll}
\text { Minimize } & \sum_{j=1}^{r} f_{j}\left(\alpha_{j}\right) \\
\text { subject to } & \sum_{j=1}^{r} g_{j}\left(\alpha_{j}\right) \leq C_{r} \\
\text { and } & \alpha_{j} \geq 0 ; j=1,2, \ldots, r
\end{array}
$$

where $f_{j}\left(\alpha_{j}\right)=\sum_{h \in I_{j}}^{r} \frac{A_{h}}{\alpha_{j} \beta_{h}}, g_{j}\left(\alpha_{j}\right)=\sum_{h \in I_{j}}^{r} \alpha_{j} c_{h} \beta_{h} \leq C_{0} ; j=1,2, \ldots, r . \mathrm{C}_{\mathrm{r}}<\mathrm{C}_{0}$ is the available budget for measurements of the selected units from the first $r$ groups. With the above definition of $\mathrm{C}_{\mathrm{r}}$ we have

$$
C_{r}=C_{0} \text { for } r=k
$$

also

$$
\begin{aligned}
& C_{r}=g_{1}\left(\alpha_{1}\right)+g_{2}\left(\alpha_{2}\right)+\ldots+g_{r}\left(\alpha_{r}\right) \\
& C_{r-1}=g_{1}\left(\alpha_{1}\right)+g_{2}\left(\alpha_{2}\right)+\ldots+g_{r-1}\left(\alpha_{r-1}\right)=C_{r}-g_{r}\left(\alpha_{r}\right) \\
& : \quad \quad \quad \quad \quad \\
& : \quad \vdots \\
& C_{2}=g_{1}\left(\alpha_{1}\right)+g_{2}\left(\alpha_{2}\right)=C_{3}-g_{3}\left(\alpha_{3}\right)
\end{aligned}
$$

and $\quad C_{1}=g_{1}\left(\alpha_{1}\right)=C_{2}-g_{2}\left(\alpha_{2}\right)$

If $f\left(r, C_{r}\right)$ denotes the minimum value of the objective function of sub problem (2.8), then

$$
f\left(r, C_{r}\right)=\underset{\text { feasible } \alpha_{j}}{\operatorname{Min}}\left\{\sum_{j=1}^{r} f_{j}\left(\alpha_{j}\right): \sum_{j=1}^{r} g_{j}\left(\alpha_{j}\right)=C_{r} \text { and } \alpha_{j} \geq 0 ; j=1,2, \ldots, r\right\}
$$

For the first stage $(\mathrm{r}=1)$

$$
f\left(1, C_{1}\right)=\frac{\left(\sum_{h \in I_{1}} \frac{A_{h}}{\beta_{h}}\right)\left(\sum_{h \in I_{1}} c_{h} \beta_{h}\right)}{C_{1}} \text { at } \alpha_{1}=\frac{C_{1}}{\sum_{h \in I_{1}} c_{h} \beta_{h}}
$$

and for $\mathrm{r} \geq 2$

$$
f\left(r, C_{r}\right)=\min _{0 \leq g_{r}\left(\alpha_{r}\right) \leq C_{r}}\left\{\frac{\sum_{h \in I_{r}} \frac{A_{h}}{\beta_{h}}}{\alpha_{r}}+f\left(r-1, C_{r}-g_{r}\left(\alpha_{r}\right)\right)\right\}
$$

Expression (2.11) gives the required recurrence relation. 
From $f(k, C)$ the optimum value of $\alpha_{k}$ is obtained, from $f\left(k-1, C-g_{k}\left(\alpha_{k}\right)\right)$ the optimum value of $\alpha_{k-1}$ is obtained and so on until $\alpha_{1}$ is determined.

After obtaining $\alpha_{j} ; j=1,2, \ldots, k$ the values of $n_{h}$ are obtained by using (1.4).

$$
V_{\text {mixed }}=\sum_{l=1}^{p} a_{l} \sum_{j=1}^{k} \sum_{h \in I_{j}} \frac{W_{h}^{2} S_{l h}^{2}}{\alpha_{j} \beta_{h}}
$$

\section{Criterion for Using Compromise Mixed Allocation}

The relative loss of efficiency (R. L. E.) by using different allocations in different groups of strata instead of optimum allocation is

$$
\text { R. L. E. }=L(\underline{n})=\frac{V_{\text {mixed }}-V_{\text {opt }}}{V_{\text {opt }}} \times 100 \%
$$

where $\underline{n}=\left(n_{1}, n_{2}, \ldots, n_{L}\right)$ is the vector of compromise mixed allocation.

$L(\underline{n})$ given by (3.1) will be the sum of the losses of the efficiencies incurred due to various allocations in different groups of strata. If any particular allocation results in a significant loss of efficiency, it may be replaced by any other more efficient allocation.

\section{A Numerical Illustration}

[4] gave a numerical illustration using artificial data. We added another characteristics to that data with the corresponding values of $s_{h}$ as, $s_{1 h}$ and $s_{2 h}$. Thus we have the following situation.

In stratification with seven strata and two characteristics the values of $N_{h}, s_{1 h}, s_{2 h}$ and $c_{h}$ are given in Table 1. It is assumed that the total available budget of the survey $C=4500$ units, which includes an overhead cost $c_{0}=500$ units. This gives the total available amount for measurements

$$
C_{0}=C-c_{0}=4500-500=4000 \text { units. }
$$

Table 1. Data for seven strata and two characteristics

\begin{tabular}{cccccc}
\hline$h$ & $N_{h}$ & $s_{1 h}$ & $s_{2 h}$ & $c_{h}$ & $W_{h}$ \\
\hline 1 & 472 & 5.237 & 7.815 & 6 & 0.1888 \\
2 & 559 & 5.821 & 7.949 & 8 & 0.2236 \\
3 & 425 & 5.238 & 7.725 & 7 & 0.1700 \\
4 & 218 & 25.528 & 30.125 & 12 & 0.0872 \\
5 & 233 & 22.232 & 32.231 & 11 & 0.0932 \\
6 & 328 & 15.129 & 18.455 & 10 & 0.1312 \\
7 & 265 & 40.125 & 45.358 & 15 & 0.1060 \\
\hline
\end{tabular}

The strata are so numbered that:

(i) Strata 1, 2 and 3 constitute group $G_{1}$ in which equal allocation is to be used, that is $\beta_{h}=1 ; h \in I_{1}=\{1,2,3\}$

(ii) Strata 4 and 5 constitute group $G_{2}$ in which proportional allocation is to be used, that is $\beta_{h}=W_{h} ; h \in I_{2}=\{4,5\}$

(iii) Strata 6 and 7 constitute group $G_{3}$ in which optimum allocation is to be used, that is $\beta_{h}=\sqrt{A_{h} / c_{h}} ; h \in I_{3}=\{6,7\}$

Thus $I_{1}=\{1,2,3\}, I_{2}=\{4,5\}$ and $I_{3}=\{6,7\}$.

It can be seen that $I_{j} ; j=1,2,3$ are mutually exclusive and exhaustive. 
It is also assumed that both the characteristics are equally important that is $a_{1}=a_{2}=0.5$.

Table 2. Values of $A_{h}, A_{h} / \beta_{h}$ and $c_{h} \beta_{h}$

\begin{tabular}{|c|c|c|c|c|c|c|c|c|c|}
\hline$h$ & $N_{h}$ & $s_{1 h}$ & $s_{2 h}$ & $c_{h}$ & $W_{h}$ & $A_{h}$ & $\beta_{h}$ & $A_{h} / \beta_{h}$ & $c_{h} \beta_{h}$ \\
\hline 1 & 472 & 5.237 & 7.815 & 6 & 0.1888 & 1.5773 & 1 & 1.5773 & 6 \\
\hline 2 & 559 & 5.821 & 7.949 & 8 & 0.2236 & 2.4266 & 1 & 2.4266 & 8 \\
\hline 3 & 425 & 5.238 & 7.725 & 7 & 0.1700 & 1.2588 & 1 & 1.2588 & 7 \\
\hline \multicolumn{7}{|c|}{$h \in I_{1}$} \\
\hline 4 & 218 & 25.528 & 30.125 & 12 & 0.0872 & 5.92793 & 0.0872 & 67.981 & 1.046 \\
\hline 5 & 233 & 22.232 & 32.231 & 11 & 0.0932 & 6.65843 & 0.0932 & 71.442 & 1.026 \\
\hline \multicolumn{7}{|c|}{$h \in I_{2}$} \\
\hline 6 & 328 & 15.129 & 18.455 & 10 & 0.1312 & 4.901 & 0.7001 & 7.0008 & 7.00 \\
\hline 7 & 265 & 40.125 & 45.358 & 15 & 0.1060 & 20.600 & 1.1720 & 17.5796 & 17.58 \\
\hline
\end{tabular}

$\left.\begin{array}{ll}\text { Minimize } & \frac{5.2627}{\alpha_{1}}+\frac{139.423}{\alpha_{2}}+\frac{24.5804}{\alpha_{3}} \\ \text { subject to } & 21 \alpha_{1}+2.072 \alpha_{2}+24.58 \alpha_{3} \leq 4000 \\ \text { and } & \alpha_{j} \geq 0 ; j=1,2,3\end{array}\right\}$

Using (2.10)

$$
f\left(1, C_{1}\right)=\frac{21 \times 5.2627}{C_{1}}=\frac{110.5167}{C_{1}} \text { at } \alpha_{1}=\frac{C_{1}}{21}
$$

Using (2.11)

$$
f\left(2, C_{2}\right)=\frac{139.423}{\alpha_{2}}+\frac{110.5167}{C_{2}-2.072 \alpha_{2}}
$$

Minimizing $f\left(2, C_{2}\right)$ with respect to $\alpha_{2}$ by differentiating and equating to zero we get:

$$
\frac{139.423}{\alpha_{2}^{2}}=\frac{110.5167 \times 2.072}{\left(C_{2}-2.072 \alpha_{2}\right)^{2}}
$$

This gives $\alpha_{2}=0.2982 C_{2}$ and $f\left(2, C_{2}\right)=\frac{756.7612}{C_{2}}$

Using recurrence relation (2.11)

$$
\begin{gathered}
f\left(3, C_{3}\right)=\frac{24.5804}{\alpha_{3}}+\frac{756.7612}{C_{3}-24.58 \alpha_{3}} \\
\Rightarrow \quad \frac{24.5804}{\alpha_{3}^{2}}=\frac{756.7612 \times 24.58}{\left(C_{3}-24.58 \alpha_{3}\right)^{2}}
\end{gathered}
$$

or $\quad \alpha_{3}=0.0192 C_{3}$

Thus $f\left(3, C_{3}\right)=\frac{2713.3153}{C_{3}}$

Putting $C_{3}=C_{0}=4000$ we get

$$
\alpha_{3}=0.0192 C_{3}=76.8
$$


Thus $\quad C_{2}=C_{3}-24.58 \alpha_{3}=2112.256$

and $\quad \alpha_{2}=0.2982 C_{2}=629.8747$

Finally $C_{1}=C_{2}-2.072 \alpha_{2}=807.1556$

and $\alpha_{1}=\frac{C_{1}}{21}=38.4360$

Using (1.4) the values of $n_{h}$ are obtained as

$$
\begin{aligned}
& n_{1}=\alpha_{1} \quad \beta_{1}=38.4360 \times 1.00 \approx 38 \\
& n_{2}=\alpha_{1} \quad \beta_{2}=38.4360 \times 1.00 \approx 38 \\
& n_{3}=\alpha_{1} \quad \beta_{3}=38.4360 \times 1.00 \approx 38 \\
& n_{4}=\alpha_{2} \quad \beta_{4}=629.8747 \times 0.0872=54.9251 \approx 55 \\
& n_{5}=\alpha_{2} \quad \beta_{5}=629.8747 \times 0.0932=58.7043 \approx 59 \\
& n_{6}=\alpha_{3} \quad \beta_{6}=76.8 \times 0.7001=53.7677 \approx 54 \\
& n_{7}=\alpha_{3} \quad \beta_{7}=76.8 \times 1.172=90.0096 \approx 90
\end{aligned}
$$

Table 3. Computation of $\alpha_{j}$

\begin{tabular}{|c|c|c|c|c|c|}
\hline \multirow{2}{*}{$\begin{array}{c}\text { Group No. } \\
j\end{array}$} & $(A)$ & $(B)$ & $(C)$ & $(D)$ & \multirow{2}{*}{$\alpha_{j}=4000 \frac{(C)}{\sum(D)}$} \\
\cline { 2 - 5 } & $\sum_{h \in I_{j}} A_{h} / \beta_{h}$ & $\sum_{h \in I_{j}} c_{h} \beta_{h}$ & $\sqrt{(A) /(B)}$ & $\sqrt{(A)(B)}$ & \\
\hline 1 & 5.2627 & 21 & 0.5006 & 10.5127 & 38.4424 \\
\hline 2 & 139.4226 & 2.0716 & 8.2038 & 16.9949 & 629.9918 \\
\hline 3 & 24.5804 & 24.5810 & 1.0000 & 24.5807 & 76.7927 \\
\hline
\end{tabular}

The computations are shown in Tables 2 and 3 .

With the values of $\alpha_{j} ; j=1,2,3$ given in last column of Table 3 , the mixed allocation is obtained as:

For $j=1 \quad n_{1(m)}=n_{2(m)}=n_{3(m)}=\alpha_{1}=38.4424$

For $j=2 \quad n_{4(m)}=\alpha_{2} \beta_{4}=\alpha_{2} W_{4}=629.9918 \times 0.0872=54.9353$

$$
n_{5(m)}=\alpha_{2} \beta_{5}=\alpha_{2} W_{5}=629.9918 \times 0.0932=58.7152
$$

For $j=3 \quad n_{6(m)}=\alpha_{3} \beta_{6}=\alpha_{3}\left(\sqrt{A_{6} / c_{6}}\right)=76.7927 \times 0.7001=53.7626$

$$
n_{7(m)}=\alpha_{3} \beta_{7}=\alpha_{3}\left(\sqrt{A_{7} / c_{7}}\right)=76.7927 \times 1.1720=90.0010
$$

The estimated variance under mixed allocation given by $(2.12)$ is $v_{\text {mixed }}=0.6783$

Table 4 gives the sample sizes when overall optimum allocation is used. These values are required to work out R. L. E.

Table 4. Sample sizes under over all optimum allocation

\begin{tabular}{ccccccc}
\hline$h$ & $W_{h}$ & $A_{h}$ & $c_{h}$ & $\sqrt{A_{h} / c_{h}}$ & $\sqrt{A_{h} c_{h}}$ & $n_{h(\text { opt })}$ \\
\hline 1 & 0.1888 & 1.5773 & 6 & 0.5127 & 3.0763 & 39.4205 \\
2 & 0.2236 & 2.4266 & 8 & 0.5507 & 4.4060 & 42.3422 \\
3 & 0.1700 & 1.2588 & 7 & 0.4241 & 2.9684 & 32.6082 \\
4 & 0.0872 & 5.9279 & 12 & 0.7028 & 8.4341 & 54.0369 \\
5 & 0.0932 & 6.6584 & 11 & 0.7780 & 8.5582 & 59.8189 \\
6 & 0.1312 & 4.9013 & 10 & 0.7001 & 7.0009 & 53.8293 \\
7 & 0.1060 & 20.6032 & 15 & 1.1720 & 17.5798 & 90.1128 \\
\hline
\end{tabular}


The estimated variance under optimum allocation is given by

$$
v_{\text {opt }}=\frac{\left(\sum_{h=1}^{L} \sqrt{A_{h} c_{h}}\right)^{2}}{C_{0}}=\frac{(52.0237)^{2}}{4000}=0.6766 .
$$

\section{The Performance of Compromise Mixed Allocation as Compared to Some Other Allocation}

In this Section a comparative study of the proposed compromise mixed allocation has been made with three other well known compromise allocations viz. Cochran's Average Allocation [13], Chatterjee's Compromise Allocation [10] and Compromise Allocation for "Minimizing Trace" [25]. However, these compromise allocations assume that the values of $W_{h}$ and $S_{h}^{2}$ are known for all strata.

\section{The Cochran's Average Compromise Allocation (ACA)}

The individual optimum allocations $n_{l h}^{*}$ are given by

$$
n_{l h}^{*}=\frac{C_{0} W_{h} S_{l h} / \sqrt{c_{h}}}{\sum_{h=1}^{L} W_{h} S_{l h} \sqrt{c_{h}}} ; \quad l=1,2 .
$$

The average compromise allocation $n_{h(A C A)}$ is given by

$$
n_{h(A C A)}=\frac{1}{p} \sum_{l=1}^{p} n_{l h}^{*} ; \quad h=1,2, \ldots, L .
$$

\section{Chatterjee's Compromise Allocation (CCA)}

Chatterjee's compromise allocation $n_{h(C C A)}$, obtained by minimizing the sum of the relative increases in the variances of the estimates is given as

$$
n_{h(C C A)}=\frac{C_{0} \sqrt{\sum_{l=1}^{p} n_{l h}^{* 2}}}{\sum_{h=1}^{L} c_{h} \sqrt{\sum_{l=1}^{p} n_{l h}^{* 2}}} ; \quad h=1,2, \ldots, L .
$$

\section{Sukhatme's Compromise Allocation (SCA)}

This compromise allocation $n_{h(S C A)}$, obtained by minimizing the trace of the variance-covariance matrix is given by

$$
n_{h(S C A)}=\frac{C_{0} W_{h} \sqrt{\sum_{l=1}^{p} S_{l h}^{2} / c_{h}}}{\sum_{h=1}^{L} W_{h} \sqrt{c_{h} \sum_{l=1}^{p} S_{l h}^{2}}} ; \quad h=1,2, \ldots, L .
$$

These compromise allocations, for the data used in Section 4, are worked out and listed in Table 5. 
Table 5. Various rounded off compromise allocations and the corresponding variances

\begin{tabular}{lllllllllllll}
\hline$h$ & Allocations & $n_{1}$ & $n_{2}$ & $n_{3}$ & $n_{4}$ & $n_{5}$ & $n_{6}$ & $n_{7}$ & $v_{1}$ & $v_{2}$ & Trace $=v_{1}+v_{2}$ & Cost incurred \\
\hline 1 & Cochran's & 39 & 42 & 32 & 54 & 59 & 54 & 91 & 0.52647 & 0.82826 & 1.35473 & 3996 \\
2 & Chatterjee's & 39 & 42 & 32 & 54 & 59 & 54 & 91 & 0.52647 & 0.82826 & 1.35473 & 3996 \\
3 & Minimizing Trace & 39 & 42 & 33 & 54 & 60 & 54 & 90 & 0.52671 & 0.82689 & 1.35360 & 3999 \\
4 & Proposed & 38 & 38 & 38 & 55 & 59 & 54 & 90 & 0.52800 & 0.82963 & 1.35763 & 3997 \\
\hline
\end{tabular}

Table 5 gives the rounded off values of the Cochran's, Chatterjee's, Sukhatme's and the Proposed compromise allocations, the variances $v_{1}$ and $v_{2}$ of the estimates of the two characteristics under study, the Trace $\left(v_{1}+v_{2}\right)$ and the total cost incurred. It can be seen that the proposed allocation is almost as precise as the other allocations (in terms of the 'Trace') that assume the knowledge of the true values of $W_{h}$ and $S_{h}^{2}$ for all strata. Whereas the proposed allocation may be used in this conditions is given in this manuscript.

\section{Conclusion}

Since the estimated relative loss in efficiency of the compromise mixed allocation as compared to the overall optimum allocation is

(R. L. E. $)_{\text {mixed }}=\frac{v_{\text {mixed }}-v_{o p t}}{v_{\text {opt }}} \times 100 \%=\frac{0.6783-0.6766}{0.6766} \times 100 \%=0.2513 \%$

which is very small, the proposed compromise mixed allocation may be used without any significant loss in the efficiency. In addition to the above fact the compromise mixed allocation also works well in comparison with other compromise allocations discussed in Section 5.

\section{References}

1. M. J. Ahsan, "A procedure for the problem of optimum allocation in multivariate stratified random sampling," Aligarh Bull. Math., Vol. 5-6, pp.37-42, 1976.

2. M. J. Ahsan and S. U. Khan, "Optimum allocation in multivariate stratified random sampling using prior information," J. Indian Statist. Assoc., Vol. 15, pp. 57-67, 1977.

3. M. J. Ahsan and S. U. Khan, "Optimum allocation in multivariate stratified random sampling with overhead cost," Metrika, Vol. 29, pp. 71-78, 1982.

4. M. J. Ahsan, Najmussehar and M. G. M. Khan, "Mixed allocation in stratified sampling," Aligarh Journal of Statisics, Vol. 25, pp. 87-97, 2005.

5. A. H. Ansari, Najmussehar and M. J. Ahsan, "On multiple response stratified random sampling design," International Journal of Statistical Sciences, Kolkata, India, Vol. 1, no. 1, pp. 1-11, 2009.

6. H. Aoyama, "Stratified random sampling with optimum allocation for multivariate populations," Ann. Inst. Statist. Math,Vol. 14, pp. 251-258, 1963.

7. L. G. Arvanitis and B. Afonja, "Use of the generalized variance and the gradient projection method in multivariate stratified random sampling," Biometrics, vol. 27, pp. 119-127, 1971.

8. M. D. Bankier, "Power allocations: Determining sample sizes for sub national areas," American Statistics, Vol. 42, pp. 174-177, 1988.

9. J. Bethel, "Sample allocation in multivariate surveys," Survey Methodology, Vol. 15, no. 1, pp. 47-57, 1989.

10. S. Chatterjee, "A note on optimum allocation," Scand. Actuar. J., Vol. 50, pp. 40-44, 1967.

11. S. Chatterjee, "Multivariate stratified surveys," J. Amer. Statist. Assoc., Vol. 63, pp. 530-534, 1968.

12. R. G. Clark and D. G. Steel, "Optimum allocation of sample to strata and stages with simple additional constraints," The Statistician, Vol. 49, no. 2, pp. 197-207, 2000.

13. W. G. Cochran, "Sampling Techniques," $3^{\text {rd }}$ ed., John Wiley, New York, 1977.

14. J. A. Díaz-García and L. U. Cortez, "Optimum allocation in multivariate stratified sampling: Multi-objective 
programming," Comunicación Técnica No. I-06-07/28-03-2006 (PE/CIMAT), 2006.

15. J. A. Díaz-García and L. U. Cortez, "Multi-objective optimisation for optimum allocation in multivariate stratified sampling," Survey Methodology, Vol. 34, no. 2, pp. 215-222, 2008.

16. J. L. Folks and C. E. Antle, "Optimum allocation of sampling units to the strata when there are $R$ responses of interest," J. Amer. Statist. Assoc., Vol. 60, pp. 225-233, 1965.

17. N. Jahan, M. G. M. Khan and M. J. Ahsan, "A generalized compromise allocation," J. Indian Statist. Assoc.,Vol. 32, pp. 95-101, 1994.

18. M. G. M. Khan, M. J. Ahsan and N. Jahan, "Compromise allocation in multivariate stratified sampling: An integer solution," Naval Res. Logist., Vol. 44, pp. 69-79, 1997.

19. M. G. M. Khan, E. A. Khan and M. J. Ahsan, "An optimal multivariate stratified sampling design using dynamic programming," Aust. N. Z. J. Stat., Vol. 45, no. 1, pp. 107-113, 2003.

20. A. R. Kokan and S. Khan, "Optimum allocation in multivariate surveys: An analytical solution," J. Roy. Statist. Soc., Vol. B29, no. 1, pp. 115-125, 1967.

21. M. Kozak, "On sample allocation in multivariate surveys," Comm. Statist. Simulation Comput., Vol. 35, pp. 901-910, 2006(a).

22. M. Kozak, "Multivariate sample allocation: Application of random search method," Statistics in Transition, Vol. 7, no. 4, pp. 889-900, 2006(b).

23. L. Kreienbrock, "Generalized measures of dispersion to solve the allocation problem in multivariate stratified random sampling," Comm. Statist. Theory Methods, Vol. 22, no. 1, 219-239, 1993.

24. A. Melaku, and G. Sadasivan, " $\mathrm{L}_{1}$-norm and other methods for sample allocation in multivariate stratified surveys," Comput. Statist. Data Anal., Vol. 5, no. 4, pp. 415-423, 1987.

25. P. V. Sukhatme, B. V. Sukhatme, S. Sukhatme and C. Asok, "Sampling Theory of Surveys with Applications," $3^{\text {rd }}$ ed., Iowa State University Press, Iowa, U.S.A., 1984.

26. F. Yates, "Sampling methods for Censuses and Surveys," $3^{\text {rd }}$ ed., Charles Griffin and Co. Ltd. London, 1960. 